\section{P123 NOVEL APPROACHES TO SYSTEMIC RHEUMATIC DISEASES}

Sara Salvucci, Antonella Festa, Emanuele Filippini, Lucia Manfredi, MariaGiovanna Danieli, Armando Gabrielli. Clinica Medica, Ospedali Riuniti di Ancona, Ancona, Italy

10.1136/lupus-2020-eurolupus. 167

Background Improving the quality of life is becoming a primary aim in the treatment of patients with chronic rheumatic diseases. In this regard, the management of pain and fatigue is a huge challenge. Hence, our goal is to evaluate whether a multidisciplinary integrative approach (based on yoga, mindfulness and Ayurveda massage) could lead to a quality of life improvement.

Methods Our multidisciplinary integrative approach to systemic rheumatic diseases consists of yoga sessions (120 min classes held once a week for 6 months), mindfulness sessions (60 min classes held once a week for 6 months) and 5 sessions of Ayurveda massage (60 min each). All courses take place inside our hospital. Quality of life (QoL) is measured at baseline and after 6 months by the validated Italian version of the Medical Outcome Study Short Form 36 (SF-36) questionnaire. Longterm effects will be assessed 9 months after the enrollment in all patients completing the program.

Results 35 outpatients with systemic lupus erythematosus (SLE) and systemic sclerosis (SSc) were invited to the program. From June 2019, 15 patients were enrolled (7 SLE, 8 SSC). All invited patients expressed high interest in the novel proposed approach. Logistics and distance from the hospital were the primary reasons of the inability to participate in most invited patients. Preliminary data will be completed in February 2020 .

Conclusions Although a larger cohort will have to confirm the ongoing study, we are confident that this holistic approach to rheumatic diseases has a high potential in improving the quality of life. All patients referred satisfaction for the project, mainly due to the fact that this experience has dramatically changed their perspective of the hospital: from a facility where medical tests and drug treatments are performed, to a place where they can make new friends and take care of their well-being.

\section{P124 THE IMPACT OF THE NEW AMERICAN COLLEGE OF CARDIOLOGYIAMERICAN HEART ASSOCIATION (ACCI AHA) DEFINITION OF HYPERTENSION ON ATHEROSCLEROTIC VASCULAR EVENTS IN SYSTEMIC LUPUS ERYTHEMATOSUS}

Konstantinos Tselios, Dafna D Gladman, Jiandong Su, Murray B Urowitz. University of Toronto Lupus Clinic, University Health Network, Toronto, Canada

10.1136/lupus-2020-eurolupus. 168

Background The 2017 ACC/AHA guidelines define hypertension as $\geq 130 / 80 \mathrm{mmHg}$ instead of $\geq 140 / 90 \mathrm{mmHg}$. Studies in systemic autoimmune diseases, where blood pressure (BP) is fluctuating over time, were not considered. Our aim was to assess the impact of the new definition on the prevalence and incidence of atherosclerotic vascular events (AVEs) in systemic lupus erythematosus (SLE).

Patients-Methods SLE patients with at least two years of follow-up and no previous AVEs were divided in three groups $(\geq 140 / 90 \mathrm{mmHg}, \quad 130-139 / 80-89 \mathrm{mmHg}$ and $<130 / 80$ $\mathrm{mmHg}$ ) according to the adjusted mean BP over that period.
They were followed until the first occurrence of an AVE (new onset of: angina, myocardial infarction, congestive heart failure, revascularization procedure, transient ischemic attack, stroke and cardiovascular death) or last visit. Prevalence and incidence rates of AVEs were calculated. SAS 9.4 was used for statistics; $p<0.05$ was considered significant.

Results 1532 patients satisfied the inclusion criteria $(88.1 \%$ females, mean age at baseline $36.2 \pm 14.3$ years, mean disease duration $6.1 \pm 6.3$ years). The prevalence of hypertension by the previous definition was $10.1 \%(155 / 1532)$ and with the current definition $30.7 \%$ (471/1532); the rest 1061 (69.3\%) were normotensives. After a mean follow-up of 10.8 years, there were 124 AVEs (104 non-fatal and 20 fatal). The total prevalence of AVEs was $32 / 155(20.6 \%)$ in the $\geq 140 / 90$ $\mathrm{mmHg}, 41 / 316(13 \%)$ in the $130-139 / 80-89 \mathrm{mmHg}$ and $51 /$ $1061(4.8 \%)$ in the normotensive group, respectively. The cumulative incidence was $18.9,11.5$ and 4.5 per 1000 patient-years, respectively. Similar trends (gradually decreasing incidence rates for the $\geq 140 / 90 \mathrm{mmHg}, 130-139 / 80-89$ $\mathrm{mmHg}$ and $<130 / 80 \mathrm{mmHg}$ group) were found individually for the coronary artery disease (CAD) events, cerebrovascular events and cardiovascular deaths.

Conclusions SLE patients with an adjusted mean BP of 130139/80-89 mmHg over two years developed approximately 2.5 fold more AVEs compared to the normotensives. The new definition of hypertension highlights a group of patients with a high incidence of AVEs who should be intensively targeted in order to improve cardiovascular outcomes.

\section{P125 INTERNET-BASED EXERCISE THERAPY IN PATIENTS WITH SYSTEMIC LUPUS ERYTHEMATODES - SYSTEMIC LUPUS ERYTHEMATODES EXERCISE PROGRAM (SLEEP)}

Simone Boedecker, Elmo Neuberger, Keito Philippi, Anna Hazenbiller, Arndt Weinmann, Andreas Schwarting, Simon Perikles, Julia Weinmann-Menke. Dept. of Nephrology and Rheumatology, Johannes Gutenberg University Mainz, Mainz, Germany

\subsection{6/lupus-2020-eurolupus. 169}

Background The SLEEP study examines the effect of an exercise therapy against a control group with TAU therapy (Treatment as usual) in SLE to identify the effect of regular physical activity on muscular development. Furthermore, we investigate the effect of physical activity on disease activity and especially on fatigue syndrome. Molecular biological markers such as cfDNA and exosomes also detect the degree of the prostration of the patients.

Methods Thirty SLE patients with laboratory as well as clinically stable status were included. The intervention group initially consisted of 24 patients, of which 12 were aerobically and 12 anaerobically active. In contrast, eight patients were included in a control group who did not do any sports. After a sports performance test had been performed, the patients were sent to a weekly training plan for a total of 12 weeks. The training was individually adapted to their performance, via an Internet platform. At the end of the week, the training plan for the following week was developed according to the patient feedback and its evaluation.

Results First evaluations show laboratory chemically stable humoral systemic activity (ANA, dsDNA-Ak, C3c, C4). Twothird of the patients reported a reduction in fatigue and a significant improvement in physical fitness. With regard to the sports medical examination before and after the 3-month training program, a significant increase in VO2peak $(p<0.001)$ 
was observed. In addition, a significant increase in muscle strength and a reduction in body fat could be demonstrated.

Conclusion Due to the initial significantly reduced cardiorespiratory fitness of the patients, but with promising first data showing a benefit of the patients after the training program, a follow-up study with a larger SLE patient collective over a period of 2 years is planned. In addition, a murine comparative study will be initiated in the spontaneous lupus mouse model of the MRL-Fas lpr mice. On the one hand, the influence of physical exercise on disease activity and progression of SLE should be analysed. Furthermore, we want to investigate the effects of physical activity on the musculature (inflammation, necrosis and fibrosis) and cardiovascular damage.

\section{P126 TOLERABILITY, EFFICACY AND ADHERENCE: WHAT DO LUPUS PATIENTS THINK ABOUT TREATMENT?}

Elvira Chocano Navarro, George Robinson, Lucia Martin Gutierrez, Thomas McDonnell, Kirsty Waddington, Elizabeth Jury, Coziana Ciurtin, Chris Wincup. Dept. of Rheumatology, University College London, London, UK

\subsection{6/lupus-2020-eurolupus. 170}

Background The treatment of systemic lupus erythematosus (SLE) can be challenging due to the complex nature of symptoms, yet despite recent advances in treatment, patients' opinion and medication beliefs are poorly understood. Furthermore, patient understanding of treatment plays a major role in medication adherence. In this study we aimed to understand patient experiences relating to treatment.

Methods Thirteen patients attended a patient engagement event. They were asked to answer 12 structured treatmentbased questions designed to assess medication beliefs. Round table discussions were held where patients shared their experiences to allow for both qualitative and quantitative data collection.

Results The majority of patients (74.5\%) believed that they had a good understanding of their current treatment with many accessing medication-related information though consultation with their rheumatologist or specialist nurse (93.3\%),

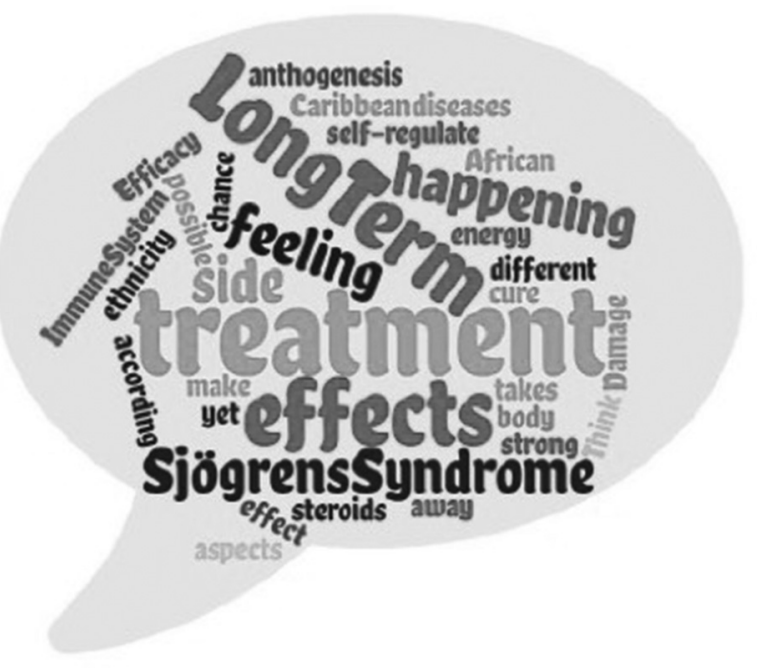

Abstract P126 Figure 1 'Word Cloud' Analysis of patient concerns prior to starting treatment

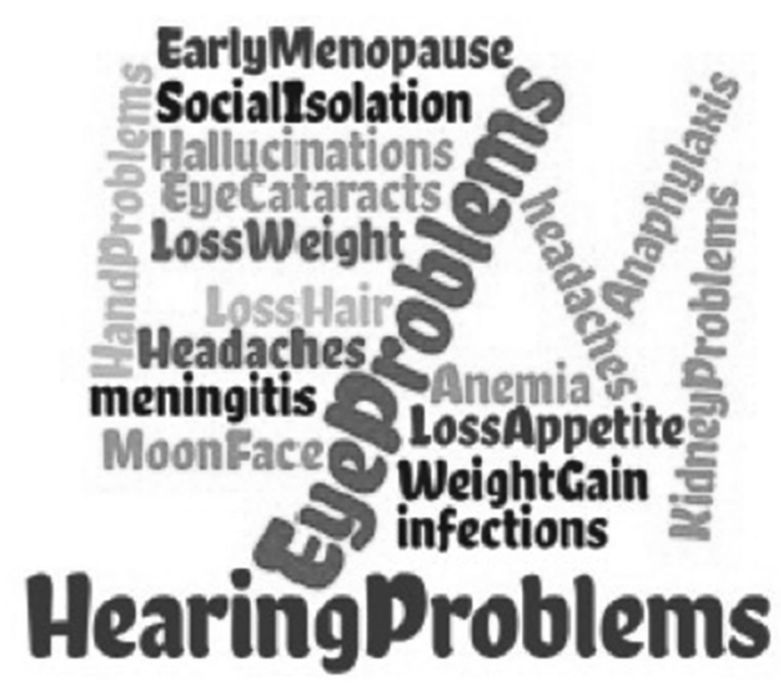

Abstract P126 Figure 2 'Word Cloud' Analysis of perceived side effects experienced by patients

via patient information sheets (33\%) and by using online resources $(46.6 \%)$.

Many patients (76.9\%) reported that they had concerns before starting a new treatment, in particular in relation to potential side effects and ultimate treatment duration. A substantial number $(75 \%)$ of patients reported a previous adverse reaction or side effect to treatment. Just over half of participants $(54.6 \%)$ were previously advised by their doctor to stop taking a medication because it was not effective. Interestingly, 40\% said they had stopped taking their medication on their own accord, usually due to side effects, with $54.5 \%$ reported stopping treatment due to feeling it to be ineffective. Figure 1 shows 'World Cloud' qualitative analysis of patient responses regarding concerns prior to starting treatment. Figure 2 shows perceived side effects reported by participants. Over 90\% of respondents thought more research should be performed in investigating potential side effects and to identify those who will respond well prior to starting a treatment.

Conclusions In summary this work shows that patients are concerned about adverse events associated with treatment and supports new research to identify ways to mitigate those effects.

\section{P127 LONG-TERM EFFECTS OF COMBINED B-CELL IMMUNOMODULATION WITH RITUXIMAB AND BELIMUMAB IN SEVERE, REFRACTORY SLE: TWO YEAR RESULTS RITUXIMAB AND BELIMUMAB COMBINATION FOR SEVERE SLE}

${ }^{1}$ Tineke Kraaij, ${ }^{1}$ Eline J Arends, 'Laura S van Dam, 'Sylvia WA Kamerling, ${ }^{2}$ Paul LA van Daele, 'Obbo W Bredewold, 'Argho Ray, ${ }^{3}$ Jaap A Bakker, ${ }^{4}$ Hans U Scherer, ${ }^{4}$ Tom JW Huizinga, ${ }^{1}$ Ton J Rabelink, ${ }^{1}$ Cees van Kooten, ${ }^{1} Y$ K Onno Teng. 'Dept of Nephrology, LUMC, Leiden; ${ }^{2}$ Dept of Clinical Immunology, Erasmus MC, Rotterdam; ${ }^{3}$ Dept of Clinical Chemistry and Laboratory Medicine, LUMC, Leiden; ${ }^{4}$ Dept of Rheumatology, LUMC, Leiden, The Netherlands

\subsection{6/lupus-2020-eurolupus. 171}

Background Anti-CD20 B-cell depletion has not shown superior efficacy to standard immunosuppression in patients with systemic lupus erythematosus (SLE). Besides trial design, 